Editorial

\title{
Microplastics-A New Journal on the Environmental Challenges and Adverse Health Effects of Microplastics
}

\author{
Nicolas Kalogerakis
}

check for

updates

Citation: Kalogerakis, N.

Microplastics-A New Journal on the Environmental Challenges and

Adverse Health Effects of

Microplastics. Microplastics 2022, 1,

1-2. https://doi.org/10.3390/

microplastics1010001

Received: 13 July 2021

Accepted: 21 July 2021

Published: 23 July 2021

Publisher's Note: MDPI stays neutral with regard to jurisdictional claims in published maps and institutional affiliations.
School of Chemical and Environmental Engineering, Technical University of Crete, 73100 Chania, Greece; nicolas.kalogerakis@enveng.tuc.gr

Microplastics is a new, open-access, peer-reviewed journal by MDPI that will provide an advanced forum for scientists to discuss the challenges faced by the ubiquitous presence of microplastics in the environment, as well as their impact on human health and ecosystem services. Microplastics (MPs) have been found in all environmental compartments such as seawater and freshwater bodies, municipal wastewater and urban runoff, agricultural soils, sediments, and air. The level of plastic debris, mainly as microplastics, in the environment is reaching unprecedented levels and there is worldwide concern about their adverse effects on both living organisms and the environment.

MPs are less than $5 \mathrm{~mm}$ in size and can be either primary, i.e., manufactured at such sizes and used as industrial feedstock material and/or in various consumer products, or secondary, i.e., resulting from the fragmentation of larger pieces of plastic due to weathering processes. Fragmentation may also yield nanoplastics, i.e., smaller fragments with characteristic sizes less than $1 \mu \mathrm{m}$, which are of a greater concern to human health than MPs are.

This journal will publish critical reviews, regular research articles, and short communications, and will host special issues on areas of increased importance and relevance to primary and secondary MPs, including nanoplastics. There are significant knowledge gaps that research in the coming years is expected to fill and lead to sustainable mitigation strategies to combat the adverse effects of MPs.

The new Microplastics journal is anticipated to tackle, among others, the following challenges:

- Fate of microplastics in the environment, including monitoring, transport modeling, and effects/impacts in various environmental compartments.

- Detection methods, including the development of reliable and validated techniques for MPs characterization and quantification, and in particular, the development or improvement of MP detection methods in complex environmental matrices.

- Microplastics as carriers for hydrophobic chemicals, heavy metals, and antibiotic resistance genes. Ramifications of the MPs and adsorbed chemicals, if ingested, on animal and human health. The effect of such environmental contaminants on accumulation, degradation, transfer, and toxicity to living organisms needs to be further investigated.

- Human exposure to microplastics and nanoplastics through ingestion of food, drinking water, and inhalation of airborne particulates results in adverse health effects that need further investigation.

- Biodegradation of microplastics and nanoplastics is an important mechanism to determine their environmental fate. Emphasis should be given to the biodegradation of MPs, which are -C-C- backbone or biodegradable polymers. In addition, technologies for treating thermosets need to be examined.

- Modeling the behavior of MPs in the environment from generation to mineralization or sequestration or ingestion, including global transport modeling of plastic fragments. 
Our aim is to publish original articles covering all aspects related to microplastics: their sources, sinks, and environmental fate; potential effects on ecosystem services and human life; and mitigation measures to reduce their adverse effects.

Conflicts of Interest: The author declares no conflict of interest.

Short Biography of Author

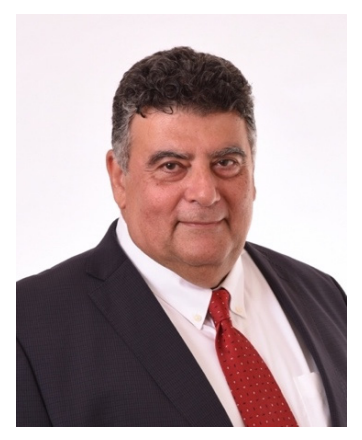

Nicolas Kalogerakis is a Professor of Biochemical Engineering at the Technical University of Crete (Greece), where he has served as Vice-President of the University Council and twice as Department Head. Prior to that, he was a Professor at SUNY-Buffalo (USA) and at the University of Calgary (Canada). He holds a Diploma in Chemical Engineering from the National Technical University of Athens, an MEng from McGill University, and a PhD from the University of Toronto.

His area of expertise includes environmental biotechnology focusing on bioremediation and phytoremediation technologies for the restoration of contaminated sites; protection and restoration of the marine environment focusing on oil spills and plastics/microplastics; novel oxygenation systems; and agricultural wastewater treatment. Currently, his group is participating in three H2020 EU-funded research projects and he is the coordinator of an ERA-MIN2 project. He was also the coordinator of the FP7-project, KILL•SPILL, that aimed to combat marine oil spills.

Prof. Kalogerakis' publication record includes six patents, one book, 216 articles in referred journals, and more than 180 presentations at international conferences. He has been an invited speaker in several conferences and academic institutions worldwide. He has over 14,700 citations and an H-index of 66 (Google Scholar, July 2021). He has served as a member of the European Commission Environment Committee (2007-2011) and as Sherpa at the European Commission High Level Group on Key Enabling Technologies (2013-2015). Finally, he is a member of the advisory board of several research institutes worldwide, and he was the recipient of the TU-Crete Research Excellence award (2017). 